\title{
Diffusion under irradiation of rare earth elements in apatite.
}

\author{
P. Martin ${ }^{a}$, A. Chevarier ${ }^{a}$, G. Panczer ${ }^{b}$ \\ INN \\ a Institut de Physique Nucléaire de Lyon', UMR 5822, IN2P3-CNRS ,Université Claude \\ Bernard - Lyon1, 43 Bd du 11 novembre 1918, F-69622, Villeurbanne Cedex, France
}

${ }^{\mathrm{b}}$ Laboratoire Physico-chimie des matériaux luminescents, UMR 5620 CNRS, Université

Claude Bernard Lyon 1, 43 Bd du 11 novembre 1918, F-69622, Villeurbanne Cedex, France

$$
\text { Lepor, } \angle \text { COM L }
$$

\begin{abstract}
Nuclear waste ceramic forms among which the apatite are under development as an alternative to waste glass in case of selective confinement. In that context, we studied the diffusion of lanthanide ions $\left(\mathrm{La}^{3+}, \mathrm{Eu}^{3+}\right)$ in hydroxyapatite over a temperature range of storage interest, taking into account a possible enhanced diffusion due to irradiation effects. The lanthanide ions are introduced in apatite targets using ion implantation. The diffusion coefficients are deduced from Rutherford Backscattering Spectroscopy at each step of annealing and irradiation procedure. Evidence of enhanced diffusion is shown and can be explained as a diffusion process governed by defect migration towards the surface. Time Resolved Laser Induced Fluorescence measurements show that, during enhanced diffusion performed under vacuum, the europium ions substitute the calcium ions preferentially in $\mathrm{Ca}(\mathrm{I})$ hydroxyapatite sites.
\end{abstract}




\section{Introduction}

Apatites are potentially valuable inertial matrices to actinide and some long lived fission products confinement[1]. Moreover, hydroxyapatite is formed as waste ashes from nuclear fuel reprocessing are incorporated in cement [2], then in this process hydroxyapatite is part of the waste matrix. The general formula for apatite is $\mathrm{Ca}_{10}\left(\mathrm{PO}_{4}\right)_{6} \mathrm{X}_{2}, \mathrm{X}$ being a fluorine, chlorine ion or an hydroxyl group. The crystal structure of apatite (space group $\mathrm{P}_{3 / \mathrm{m}}$ ) permits a wide range of cation and anion substitutions [3][4][5]. In particular the two calcium positions have distinct stereochemistries $\left(\mathrm{Ca}(\mathrm{I})\right.$ with $\mathrm{C}_{3}$ symmetry surrounded by 9 oxygen and $\mathrm{Ca}(\mathrm{II})$ with $\mathrm{C}_{\mathrm{s}}$ symmetry surrounded by 6 oxygen and $1\left(\mathrm{OH}^{-}\right)$ion [6][7]). They able to accommodate a variety of univalent, divalent, and trivalent cations as substituents [8].

The purpose of this work is to study diffusion of lanthanum and europium enhanced by alpha radioactivity environment. Lanthanum has been chosen as representative of a major yield fission products. Europium is representative of actinides as the chemical properties of $\mathrm{Eu}^{3+}$ are very similar to those of trivalent actinides and more precisely to $\mathrm{Am}^{3+}$. Furthermore, $\mathrm{Eu}^{3+}$ can be used as a local structural probe [9]. Implantation is used to introduce $\mathrm{La}$ and $\mathrm{Eu}$ ions into apatite. During annealing procedure the samples are irradiated by bismuth ions at $100 \mathrm{keV}$ energy. These $100 \mathrm{keV} \mathrm{Bi}$ ions are representative of recoil nuclei resulting from actinide alpha radioactivity. They are known to produce a large atomic displacement rate [10]. Diffusion data are deduced from Rutherford Backscattering Spectroscopy (RBS) measurements and are analyzed in a diffusion model frame [11]. These results are discussed together with structural data obtained by Time Resolved Luminescence Spectroscopy. 


\section{Diffusion measurements}

\subsection{Experimental}

Synthetic microcrystalline hydroxyapatite referenced as DNA Grade Biogel HTP stacked into pellets at $0.4 \mathrm{GPa}$ was used. $30 \mathrm{keV} \mathrm{La}^{+}$or $\mathrm{Eu}^{+}$implantations were performed at room temperature with a dose of $5 \times 10^{15}$ at $/ \mathrm{cm}^{2}$. Afterwards four series of implanted samples were submitted to successive $\mathrm{Bi}^{2+}$ irradiation, the annealing temperature being respectively set to 25 , 150,300 and $500^{\circ} \mathrm{C}$. To understand the influence of the irradiation flux, the $\mathrm{Bi}^{2+}$ beam intensity was kept constant and equal to 5, 10 and $20 \mu \mathrm{A}$. Irradiation time was set in order to lead to cumulative doses ranging between $2.5 \times 10^{15} \mathrm{at} / \mathrm{cm}^{2}$ to $7.5 \times 10^{15} \mathrm{at} / \mathrm{cm}^{2}$. It must be notice that the bismuth ion range in the apatite pellets is equal to $40 \mathrm{~nm}$, which is much larger than the depth range corresponding to the lanthanum distribution $(16 \mathrm{~nm})$; thus there is no overlap between the two distributions. At each step of the procedure, RBS analysis was performed allowing measurements of lanthanum profiles as a function of annealing and bombardment conditions.

\subsection{Analysis}

Lanthanum profiles deduced from RBS measurements are shown figure 1 in case of $150^{\circ} \mathrm{C}$ annealing and for different $\mathrm{Bi}$ doses. We can observe a slight shift of the whole lanthanum distribution which can be explained by the monodirectional beam bombardment. These distributions are analyzed on the basis of a model derived from Fick second Law :

$$
\frac{\partial C(x, t)}{\partial t}=\frac{\partial}{\partial x}\left(D \frac{\partial C(x, t)}{\partial x}\right)-v \frac{\partial C(x, t)}{\partial x}
$$

where $C(x, t)$ is the lanthanum concentration, $D$ the diffusion coefficient and , $v$ a term of migration related to the driving force $f$ by the Nernst relation : 


$$
v=\frac{f \cdot D}{k T}
$$

Equation (1) is solved using a numerical procedure based on finite differences which allows to reproduce the lanthanum profile evolution. This approach, is fully described in $\mathrm{J}$. Cranck's book [12] and our implementation is based on the NAG FORTRAN library routine [13]. The diffusion coefficient values are deduced from the fit optimization.

Results are presented in figure 2 . In the $25-500^{\circ} \mathrm{C}$ temperature range, irradiation enhanced diffusion coefficients are nearly independent on temperature, they depend only on the bismuth beam intensity. This behavior is typical of a radiation enhanced diffusion governed by defects annihilation towards the surface. In this case, the irradiation rate is strong enough to prevent vacancy-interstitial spontaneous recombination. Radiation enhanced diffusion is described by the formalism of Dienes et al. [14], the equations are simplified as there is no vacancy-interstitial spontaneous recombination :

$$
\begin{aligned}
& \left\{\begin{array}{l}
\frac{\partial C_{\mathrm{vac}}}{\partial \mathrm{t}}=\frac{\partial}{\partial \mathrm{x}}\left(\mathrm{D}_{\mathrm{vac}} \cdot \frac{\partial \mathrm{C}_{\mathrm{vac}}}{\partial \mathrm{x}}\right)+\mathrm{P}(\mathrm{x})-\mathrm{K}_{\mathrm{vac}} \cdot\left(\mathrm{C}_{\mathrm{vac}}-\mathrm{C}_{\mathrm{vac}}^{\mathrm{th}}\right) \\
\frac{\partial \mathrm{C}_{\mathrm{int}}}{\partial \mathrm{t}}=\frac{\partial}{\partial \mathrm{x}}\left(\mathrm{D}_{\mathrm{int}} \cdot \frac{\partial \mathrm{C}_{\mathrm{int}}}{\partial \mathrm{x}}\right)+\mathrm{P}(\mathrm{x})-\mathrm{K}_{\mathrm{int}} \cdot \mathrm{C}_{\mathrm{int}}
\end{array}\right. \\
& \mathrm{D}^{*}=\mathrm{D}_{\mathrm{vac}} \cdot \mathrm{C}_{\mathrm{vac}}+\mathrm{D}_{\mathrm{int}} \cdot \mathrm{C}_{\mathrm{int}}
\end{aligned}
$$

$\mathrm{C}_{\mathrm{vac}}$ and $\mathrm{C}_{\mathrm{int}}$ are the total atomic fractions of vacancies and interstitial, $\mathrm{C}^{\text {th }}$ vac is the atomic fraction of vacancy due to thermal annealing only, $P(x)$ is the production rate of vacancy-interstitial pairs, $\mathrm{K}_{\mathrm{vac}}$ and $\mathrm{K}_{\mathrm{int}}$ are the characteristic proportionality constants for the rate of defects removal, $D_{v a c}$ and $D_{\text {int }}$ are the diffusion coefficients for vacancies and interstitials, $D^{*}$ the enhanced atomic diffusion coefficient.

In steady state, equations (3) are then given by : 


$$
\left\{\begin{array}{l}
\frac{\partial C_{\mathrm{vac}}}{\partial \mathrm{t}}=\mathrm{P}(\mathrm{x})-\mathrm{K}_{\mathrm{vac}} \cdot\left(\mathrm{C}_{\mathrm{vac}}-\mathrm{C}_{\mathrm{vac}}^{\mathrm{th}}\right)=0 \\
\frac{\partial \mathrm{C}_{\mathrm{int}}}{\partial \mathrm{t}}=\mathrm{P}(\mathrm{x})-\mathrm{K}_{\mathrm{int}} \cdot \mathrm{C}_{\mathrm{int}}=0
\end{array}\right.
$$

The constants $\mathrm{K}_{\mathrm{int}}$ and $\mathrm{K}_{\mathrm{vac}}$ are given by the relation (6):

$$
\left\{\begin{array}{l}
\mathrm{K}_{\mathrm{vac}}=\alpha_{\mathrm{vac}} \cdot v_{\mathrm{vac}} \cdot \lambda_{\mathrm{vac}}^{2} \\
\mathrm{~K}_{\mathrm{int}}=\alpha_{\mathrm{int}} \cdot v_{\mathrm{int}} \cdot \lambda_{\mathrm{int}}^{2}
\end{array}\right.
$$

Where $v_{\mathrm{vac}}$ and $v_{\text {int }}$ are the effective jump frequencies for vacancies and interstitials, $\lambda_{\text {vac }}$ and $\lambda_{\text {int }}$ the jump distances for vacancies and interstitials, $\alpha_{\text {int }}$ and $\alpha_{\text {vac }}$ the proportionality constants. The diffusion coefficients are also expressed as function of jump frequencies and jump distances by the following relations :

$$
\left\{\begin{array}{l}
D_{\mathrm{vac}}=\mathrm{C}_{\mathrm{vac}} \cdot v_{\mathrm{vac}} \cdot \lambda_{\mathrm{vac}}^{2} \\
\mathrm{D}_{\mathrm{int}}=\mathrm{C}_{\mathrm{int}} \cdot v_{\mathrm{int}} \cdot \lambda_{\mathrm{int}}^{2}
\end{array}\right.
$$

From equations (5), (6) and (7) we obtained the expressions of the diffusion coefficients :

$$
\left\{\begin{array}{l}
\mathrm{D}_{\mathrm{vac}}=\frac{\mathrm{P}(\mathrm{x})}{\alpha_{\mathrm{vac}}}+\mathrm{C}_{\mathrm{vac}}^{\mathrm{th}} \cdot v_{\mathrm{vac}} \cdot \lambda_{\mathrm{vac}}^{2} \\
\mathrm{D}_{\mathrm{int}}=\frac{\mathrm{P}(\mathrm{x})}{\alpha_{\mathrm{int}}}
\end{array}\right.
$$

As the thermal diffusion coefficient [15] $\left(\mathrm{C}^{\text {th }}{ }_{\mathrm{vac}} \cdot \mathrm{v}_{\mathrm{vac}} \cdot \lambda_{\mathrm{vac}}{ }^{2}\right)$ is negligible compared to enhanced diffusion, we see from equation (8) that $D_{\text {vac }}$ and $D_{\text {int }}$ are temperature independent.

The increased vacancy concentration due to the irradiation causes a proportional increase in diffusion by the annihilation mechanism. Then, the diffusion coefficient is proportional to the beam intensity. Such behavior is observed in figure 2, where the diffusion 
coefficients are shown to be temperature independent and to vary linearly with bismuth beam intensity :

$$
\begin{aligned}
& D_{\mathrm{I}=2.5 \mu \mathrm{A}}=(2.5 \pm 0.2) \times 10^{-15} \mathrm{~cm}^{2} / \mathrm{s}, \\
& D_{\mathrm{I}=5 \mu \mathrm{A}}=(5.2 \pm 0.7) \times 10^{-15} \mathrm{~cm}^{2} / \mathrm{s}, \\
& D_{\mathrm{I}=10 \mu \mathrm{A}}=(1.2 \pm 0.1) \times 10^{-14} \mathrm{~cm}^{2} / \mathrm{s}
\end{aligned}
$$

The question which then arises is whether such an enhanced migration is connected with lanthanide ion substitution in calcium sites.

\section{Eu location}

\subsection{Experimental}

A pulsed nitrogen laser (Molectron, $\lambda_{\text {exc. }}: 337 \mathrm{~nm}$ ) was used as an excitation source. The spectral analysis of the luminescence was achieved at room temperature, by a MS 125 Oriel monochromator (400 and 1200 lines/mm gratings) and an intensified CCD detector (LOT-ORIEL INSTASPEC V) coupled with a delay generator (Stanford RS DG535) enabling time-resolving spectra acquisition. Long decay emissions of $\mathrm{Eu}^{3+}$ ions were recorded with a delay of $1 \mu \mathrm{s}$ and a gate width of $2 \mathrm{~ms}$ while short decay emissions of $\mathrm{Eu}^{2+}$ were recorded without delay and with a gate width of $100 \mathrm{~ns}$.

\subsection{Analysis}

The emission spectra of the Eu as implanted pellet shows the characteristic bands of $\mathrm{Eu}^{3+}$. They correspond to two sets of Eu $\mathrm{Eu}^{3+}$ transitions, ${ }^{5} \mathrm{D}_{0} \rightarrow{ }^{7} \mathrm{~F}_{0},{ }^{7} \mathrm{~F}_{1},{ }^{7} \mathrm{~F}_{2}$, related to $\mathrm{Ca}$ (II) $(574,629 \mathrm{~nm})$ and $\mathrm{Ca}(\mathrm{I})$ positions $(578,591$ and $617 \mathrm{~nm})$ [9]. In figure 3 we observe that after bombardment, the first set related to $\mathrm{Ca}$ (II) substitution disappears while the second one 
related to $\mathrm{Ca}(\mathrm{I})$ substitution remains. The emission intensity of $\mathrm{Eu}^{3+}$ in $\mathrm{Ca}(\mathrm{I})$ position increases regularly with the $\mathrm{Bi}$ dose, but over $10^{16} \mathrm{Bi} / \mathrm{cm}^{2}$ the emission is quenched due to ablation effect. Along with these data, the short decay emission $(\tau=600 \mathrm{~ns})$ of $\mathrm{Eu}^{2+}$ is observed as a broad weak band $(425-460 \mathrm{~nm})$. It follows a similar evolution to that of $\mathrm{Eu}^{3+}$ but, in this case, the $\mathrm{Ca}(\mathrm{I})$ and $\mathrm{Ca}(\mathrm{II})$ contributions could not be resolved.

\section{Discussion :}

Evidence of Lanthanum ion irradiation enhanced diffusion with $100 \mathrm{keV}$ bismuth beam has been shown. It can be explained as a diffusion process governed by a defect migration towards the surface. This process has been obtained in case of bismuth doses lower than $10^{16} \mathrm{at} / \mathrm{cm}^{2}$. In such conditions, a surface sputtering phenomenon does not affect the lanthanum profile. Moreover grazing angle $\mathrm{X}$ ray diffraction shows that amorphisation of the apatite lattice is negligible. In order to get information on possible substitution of lanthanide ion in the apatite lattice, Time Resolved Laser induced Measurement has been undertaken using Eu implanted ions as a probe.

The weak emission of the Eu as implanted pellet indicates that the main part of the Eu ions is located in interstitial positions of the apatite microcrystallites. However, a small amount of Eu ions is shown to occupy the substitional sites, $\mathrm{Ca}$ (II) and $\mathrm{Ca}$ (I). Analysis of emission spectra obtained after $\mathrm{Bi}$ bombardment allows to conclude that the main mechanism is the diffusion of Eu ions from interstitials, as well as from $\mathrm{Ca}$ (II) substitutionnal positions, to the high symmetry $\mathrm{Ca}(\mathrm{I})$ site. This diffusion convergence to $\mathrm{Ca}(\mathrm{I})$ site is consistent with the fact that the bombardment was conducted under vacuum. The same behavior has been observed during Eu thermal doping under vacuum by Gaft et al. [9]. The $\mathrm{Ca}^{2+} \rightarrow \mathrm{Eu}^{3+}$ substitution can occur much more easily with charge compensation provided by vacancies 
than in the case of $\mathrm{Ca}(\mathrm{II})$. Furthermore, the non oxidizing atmosphere leads to the Eu partial reduction in $\mathrm{Eu}^{2+}$ ions, which are located in $\mathrm{Ca}$ positions in the same way as $\mathrm{Eu}^{3+}$ ions. The much broader emission band unable to determine the kind of involved Ca site. According to our previous results [15] on thermal diffusion performed under air, the reducing or oxidizing environment appears to be the key parameter for the Eu diffusion and for its substitution location inside the apatite structure.

\section{References}

[1] J. Carpena and J.L. Lacout, French patent $n^{\circ} 9307265$.

[2] E. Revertegat and G. Moine, Treatment and conditioning of radioactive incinerator ashes, Elsevier Applied Science Press, London and New York, 1991.

[3] E. Fleet and Y. Pan, J. of Solid State Chemistry, 112 (1994) 78.

[4] M.E. Fleet and Y. Pan, American Mineralogist 80 (1995) 329.

[5] M. Mikou, A. Taitai and J.L. Lacout, Ann. Chim. Fr. 10 (1985) 645.

[6] R. Jagannathan and M. Kottaisamy, J. Phys. Condens. Matter. 7 (1995) 8453.

[7] R. Knubovets, Rev. Chem. Eng. 9 (1993) 161.

[8] J. Carpena, J.L. Lacout, l'Actualité chimique 2 (1997) 3.

[9] M. Gaft, R. Reisfeld, G. Panczer, S. Shoval, B. Champagnon and G. Boulon, J. of Luminescence $72-74$ (1997) 572.

[10] R. C. Ewing, W. J. Weber and F.W. Clinard Jr., Progress in Nuclear Energy 29 (1995) 63.

[11] J. Philibert, Diffusion et transport de matières dans les solides, les éditions de Physique, Les Ulis, 1985. 
[12] J. Crank, The Mathematics of Diffusion, $2^{\text {nd }}$ Ed, Claredon Press, Oxford, 1975.

[13] NAGLIB Manual-FORTRAN, Mark 18, Vol.2, D03PCF, (1997).

[14] G.J. Dienes, A.C. Damask, J of Applied Physics, 29 (1958) 1713.

[15] P. Martin, Etude des mécanismes de diffusion des terres rares dans les apatites soumises à irradiation alpha, Lyon1 University, France, thesis, 1999. 


\section{Figure captions}

Figure 1 : Lanthanum profiles deduced from RBS measurements in case of $150^{\circ} \mathrm{C}$ annealing and after Bismuth bombardment at different doses.

Figure 2 : La diffusion coefficient vs temperature and for different bismuth beam intensities.

Figure 3 : Emission spectra of as Eu implanted and of Bi bombarded apatite $\left(\lambda_{\text {exc. }}=337 \mathrm{~nm}\right)$ 


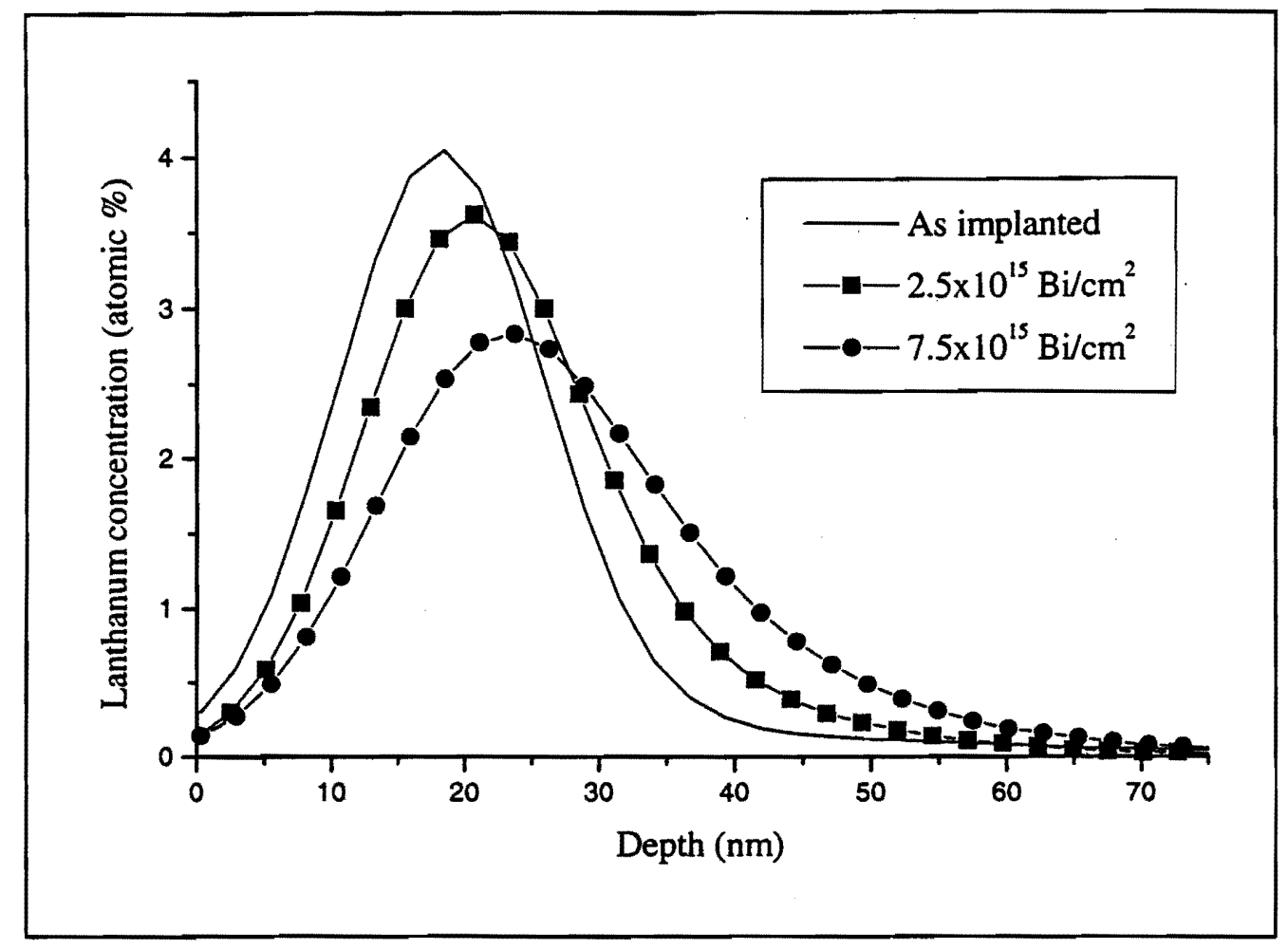

Figure 1 


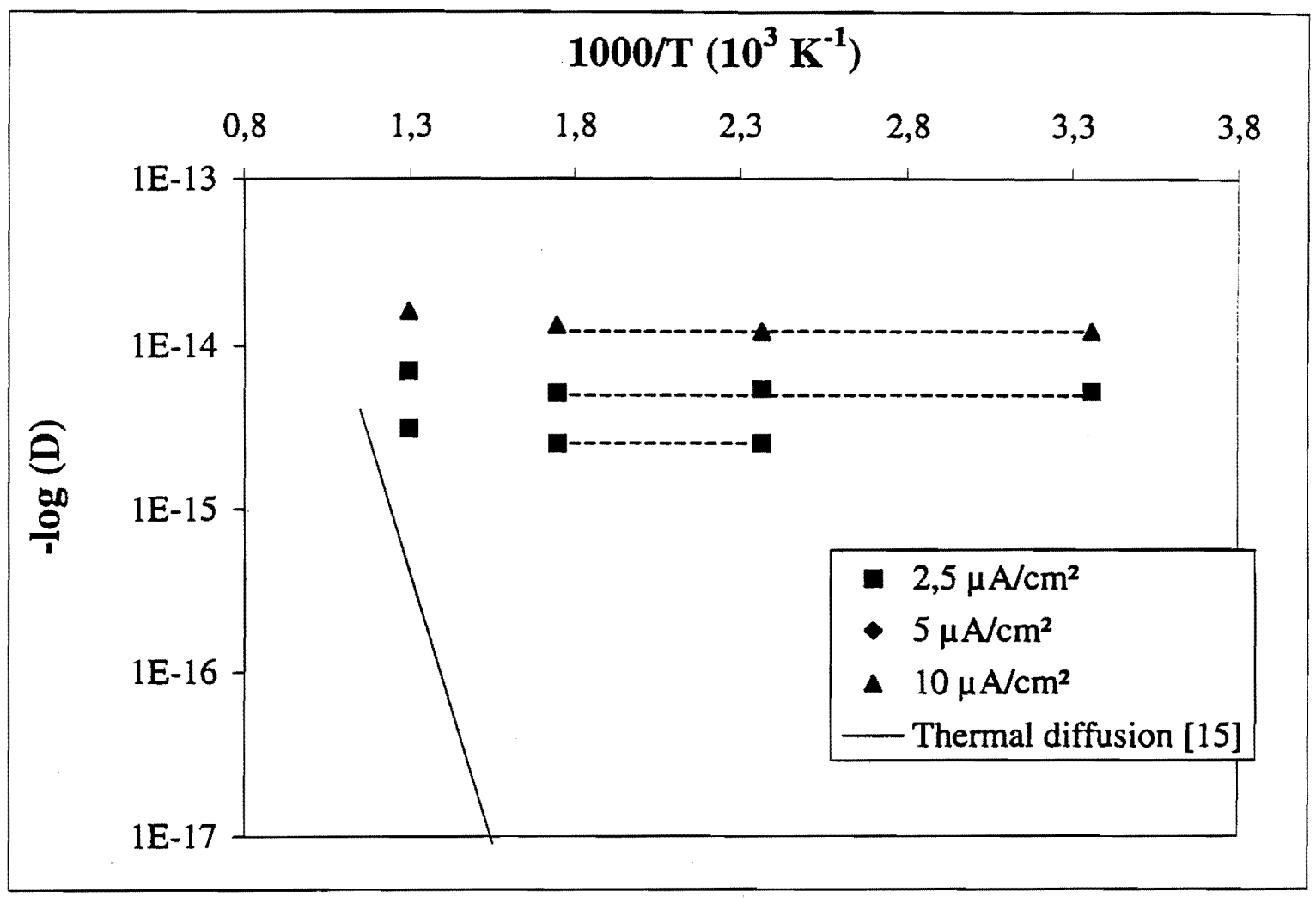

Figure 2 


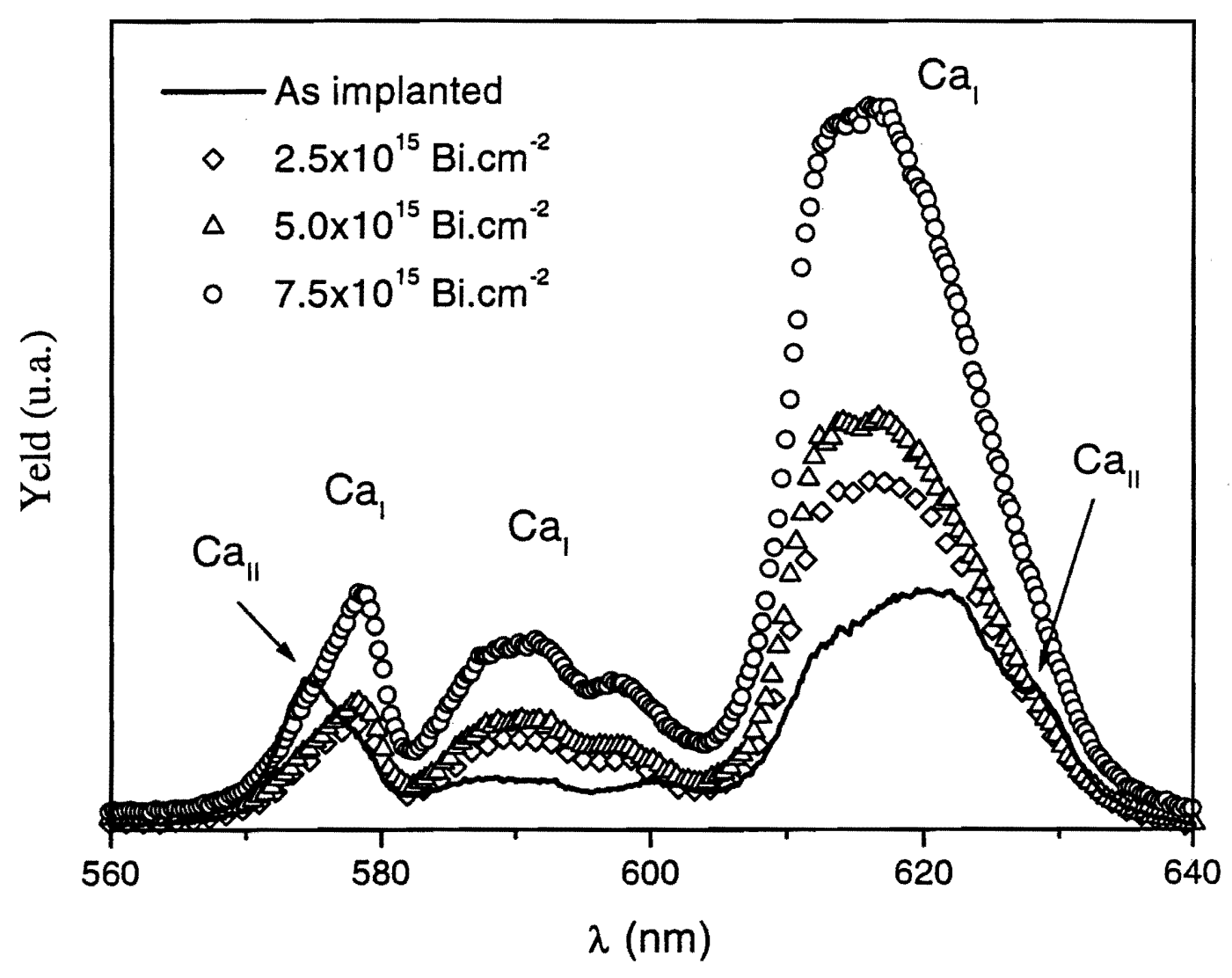

Figure 3 